\title{
Preparing silver nanoparticles in supercritical water
}

\author{
Kai Li, Fu-Shen Zhang * \\ Research Center for Eco-Environmental Sciences, Chinese Academy of Sciences, Beijing, 100085, China
}

\section{A R T I C L E I N F O}

\section{Article history:}

Received 5 September 2008

Accepted 5 November 2008

Available online 12 November 2008

\section{Keywords:}

Silver nanoparticles

Nanomaterials

Catalysts

Silver microparticles

Supercritical water

Reaction time

\begin{abstract}
A B S T R A C T
Silver (Ag) nanoparticles were prepared using Ag microparticles as precursors in supercritical water (SCW). The effect of reaction time on preparing Ag nanoparticles was investigated. The obtained products were characterized by X-ray diffraction (XRD) and transmission electron microscopy (TEM) measurements. The results indicated that, with the increase of reaction time, $\mathrm{Ag}$ microparticles prepared by calcination method with the sizes $>2 \mu \mathrm{m}$ remained after SCW treatment because of their high crystallinity, but those with the sizes $<1 \mu \mathrm{m}$ were destroyed and formed many Ag nanoparticles due to the destructive effect of SCW.
\end{abstract}

(c) 2008 Elsevier B.V. All rights reserved.

\section{Introduction}

Silver (Ag) nanoparticles have been applied in many areas because of their good surface plasma resonance (SPR), surface-enhanced Raman scattering (SERS) effects, antibacterial capability, catalytic property and high reactivity [1-3]. Up to now, many methods have been developed to prepare Ag nanoparticles. Among them, polymerprotected reduction method and microemulsion method are widely used [4-7]. Polymer-protected reduction method has been used for preparing Ag nanoparticles in the presence of polymer-protectors by reducing silver nitrate $\left(\mathrm{AgNO}_{3}\right)$. However, the size and dispersion of prepared Ag nanoparticles are greatly affected by the species and concentrations of polymer-protectors, the molar ratio of polymerprotectors to $\mathrm{AgNO}_{3}$. $\mathrm{Ag}$ nanoparticles with the sizes smaller than $30 \mathrm{~nm}$ have been successfully obtained by microemulsion method, but the process of synthesizing microemulsions is extremely complex and there are many influencing factors during the preparation of $\mathrm{Ag}$ nanoparticles.

Supercritical water (SCW) has been used in many fields of chemistry due to its unique properties such as high diffusivity, low viscosity and lack of toxicity. SCW provides an excellent reaction medium for hydrothermal synthesis of metal and metal oxide nanoparticles. Ziegler et al. reported copper (II) nitrate $\left(\mathrm{Cu}\left(\mathrm{NO}_{3}\right)_{2}\right)$ hydrolyzed to form polydisperse copper $(I)$ oxide $\left(\mathrm{Cu}_{2} \mathrm{O}\right)$ particles with diameters from 10 to $35 \mathrm{~nm}$ without alkanethiol ligands in SCW. However, in the presence of 1-hexanethiol, copper nanocrystals

\footnotetext{
* Corresponding author. Tel./fax: +86 1062849515.

E-mail address: fszhang@rcees.ac.cn (F.S. Zhang).
}

( $\mathrm{Cu}, \sim 7 \mathrm{~nm}$ in diameter) were obtained. In addition, they studied the effects of different precursors on the morphologies of prepared particles [8]. Viswanathan et al. obtained zinc oxide nanoparticles (ZnO, 39-320 $\mathrm{nm}$ ) by oxidation of zinc acetate in SCW. The effects of flow rate and feed concentration were also studied in their work [9]. To our knowledge, however, very limited literatures have reported Ag nanoparticles obtained in SCW [10]. And there are no reported studies of using Ag microparticles as precursors to prepare $\mathrm{Ag}$ nanoparticles in SCW thus far. In this paper, $\mathrm{Ag}$ microparticles were prepared by calcination method. Ag nanoparticles were subsequently obtained in SCW using these Ag microparticles as precursors. The effect of reaction time on preparing Ag nanoparticles in SCW was extensively investigated.

\section{Experimental}

\subsection{Preparation of Ag samples}

The experimental process was as follows: Firstly, $1.0 \mathrm{~g}$ of silver chloride $(\mathrm{AgCl})$ and $2.0 \mathrm{~g}$ of sodium carbonate $\left(\mathrm{Na}_{2} \mathrm{CO}_{3}\right.$, keeping in excess) were mixed homogeneously in ceramic crucible. Then the crucible was placed in a muffle and heated at $550{ }^{\circ} \mathrm{C}$ for $3 \mathrm{~h}$. After that, the muffle was cooled down to room temperature and the product was filtrated and washed with deionized water to remove the byproducts. The product was dried at $80{ }^{\circ} \mathrm{C}$ under vacuum for $2-3 \mathrm{~h}$. S-1 was obtained by this procedure.

Secondly, $2 \mathrm{ml} 0.1 \mathrm{~mol} / \mathrm{L}$ sodium hydroxide $(\mathrm{NaOH})$ solution was added to $75 \mathrm{ml}$ deionized water (the $\mathrm{pH}$ value of the obtained solution was 8.5), then $5 \mathrm{ml}$ of hydrogen peroxide $\left(\mathrm{H}_{2} \mathrm{O}_{2}\right)$ was dissolved into the solution. The mixture was stirred for a short time and transferred into a stainless steel autoclave with a capacity of $100 \mathrm{ml}$. After $0.25 \mathrm{~g}$ of 


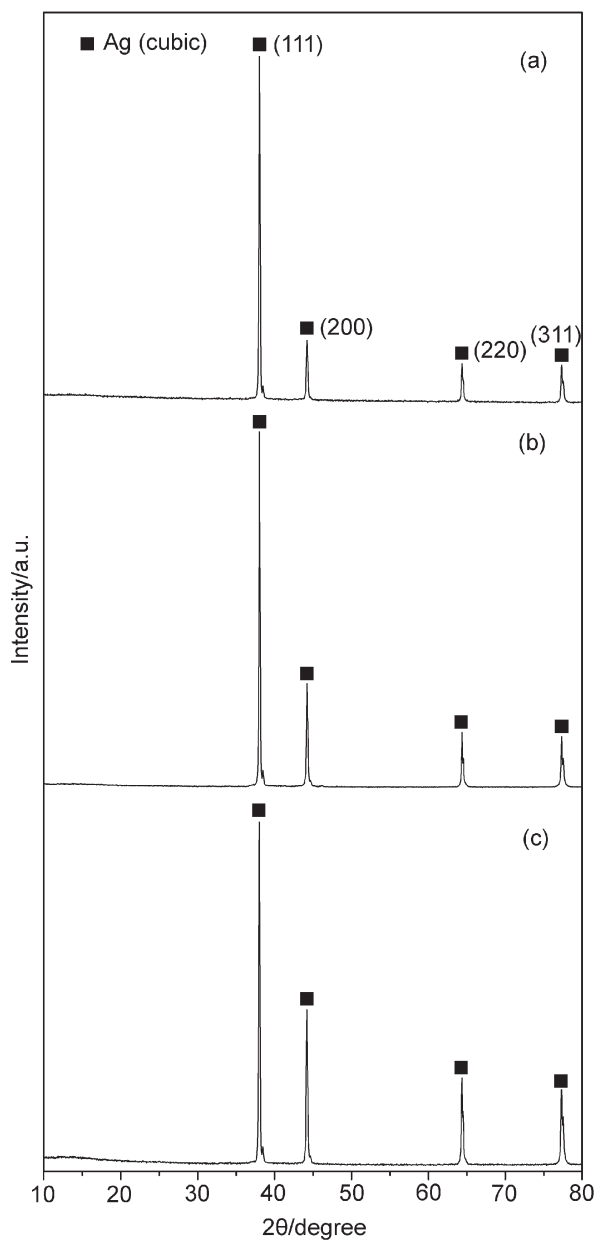

Fig. 1. XRD patterns of Ag samples (a) S-1; (b) S-2; (c) S-3.
S-1 was added into the mixture, the autoclave was sealed and heated at $450{ }^{\circ} \mathrm{C}$ for $15 \mathrm{~min}$, while the reaction pressure was kept at $33.5 \mathrm{MPa}$. The autoclave was cooled down to room temperature, and the product was filtrated and washed with deionized water. The product was then dried at $80^{\circ} \mathrm{C}$ under vacuum for $2-3 \mathrm{~h}$, and the obtained product was nominated as S-2.

In order to investigate the effect of reaction time on preparing $\mathrm{Ag}$ nanoparticles in SCW, the other parallel experiment was conducted following the same process as that of sample S-2, except that the reaction time was $30 \mathrm{~min}$. The sample thus prepared was denoted as $\mathrm{S}-3$.

\subsection{Characterization}

The phase of the samples was identified by XRD patterns, which were taken on a Philip X'pert X-ray diffractometer $(\mathrm{Cu} \mathrm{K}, V=40 \mathrm{kV}$, $I=40 \mathrm{~mA}$ ). Prior to the measurement, the samples were ground thoroughly in an agate mortar. The morphologies of the samples were taken on a Hitachi H-7500 TEM, with an accelerating voltage of $80 \mathrm{kV}$. Prior to TEM measurement, the samples were ultrasonically treated in methanol for $30 \mathrm{~min}$.

\section{Results and discussion}

XRD patterns of S-1, S-2 and S-3 are shown in Fig. 1. As demonstrated in Fig. 1, only diffraction peaks of cubic Ag can be observed, indicating that cubic Ag is the only crystalline phase in the samples.

Generally, TEM can be used to observe the morphologies of the samples. Fig. 2 illustrates the TEM images of S-1 prepared by calcination method. These particles in Fig. 2(a) and (b) are quasi-circular and the sizes of them are bigger than $2 \mu \mathrm{m}$. Furthermore, most of these microparticles aggregate together. In Fig. 2(c) and (d), there are many circular particles and the sizes of them are smaller than $1 \mu \mathrm{m}$. Similar to the large quasi-circular particles, these circular particles also aggregate together. XRD result indicates that these particles are all $\mathrm{Ag}$ microparticles.

Fig. 3 shows the TEM images of S-2 prepared in SCW for 15 min. As can be seen, there are also many large quasi-circular particles with the sizes bigger than $2 \mu \mathrm{m}$. (a)

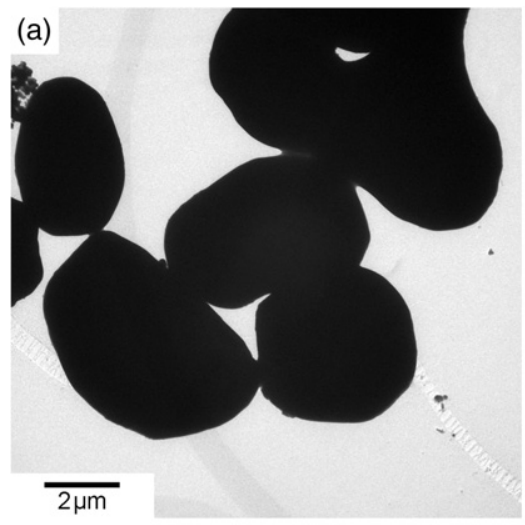

(c) (b)

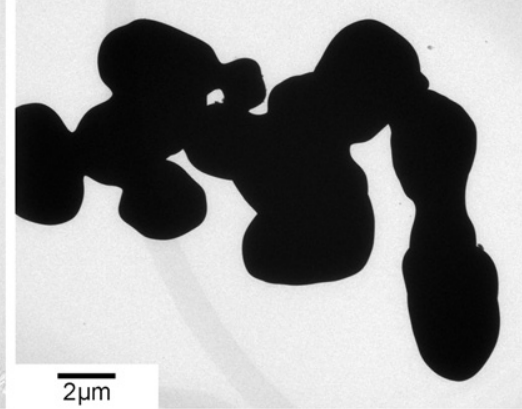

(d)

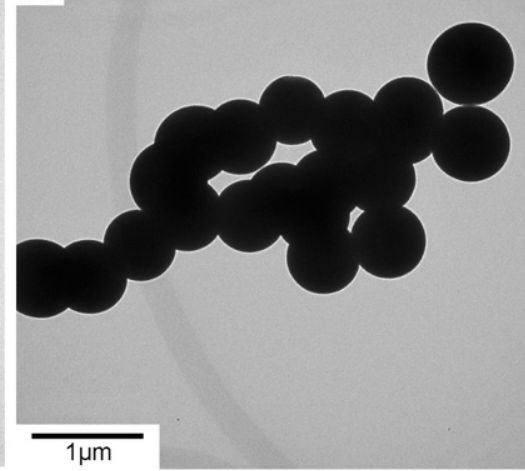

Fig. 2. TEM images of S-1 prepared by calcination method. 
(a)

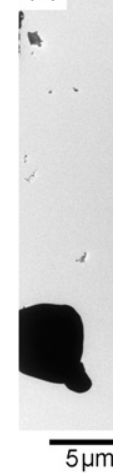

(c)
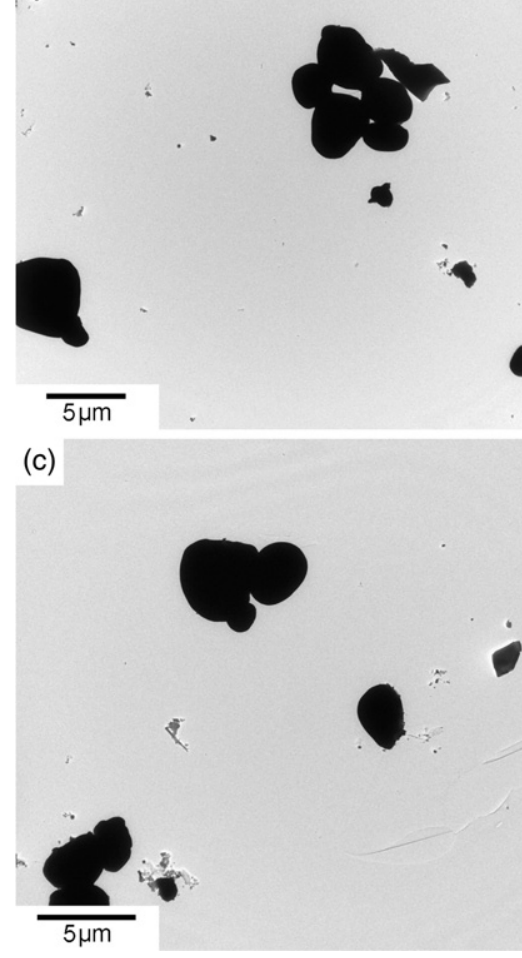

(b)

2
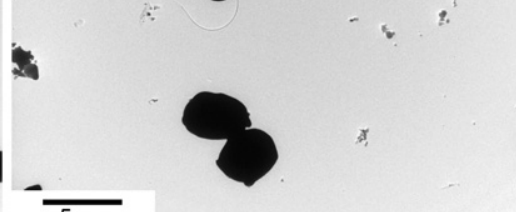

(d)

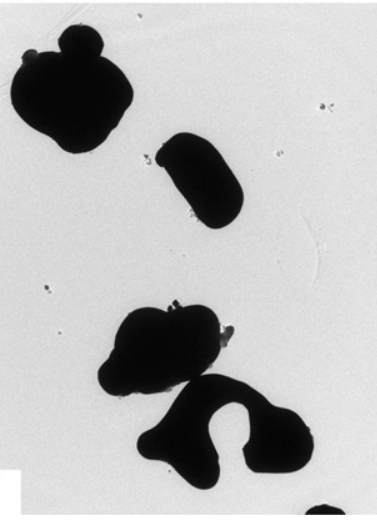

Fig. 3. TEM images of S-2 prepared in SCW for $15 \mathrm{~min}$.

Compared with Fig. 2, some small particles with irregular shapes are obtained. And the sizes of them are smaller than $1 \mu \mathrm{m}$. In addition, circular particles (shown in Fig. 2(c) and (d)) disappear in Fig. 3.
Fig. 4 illustrates the TEM images of S-3 prepared in SCW for 30 min. Similar to Fig. 3, there are many large quasi-circular particles in Fig. 4(a) and (b), and the sizes of them are bigger than $2 \mu \mathrm{m}$. These Ag microparticles also aggregate together. Circular particles
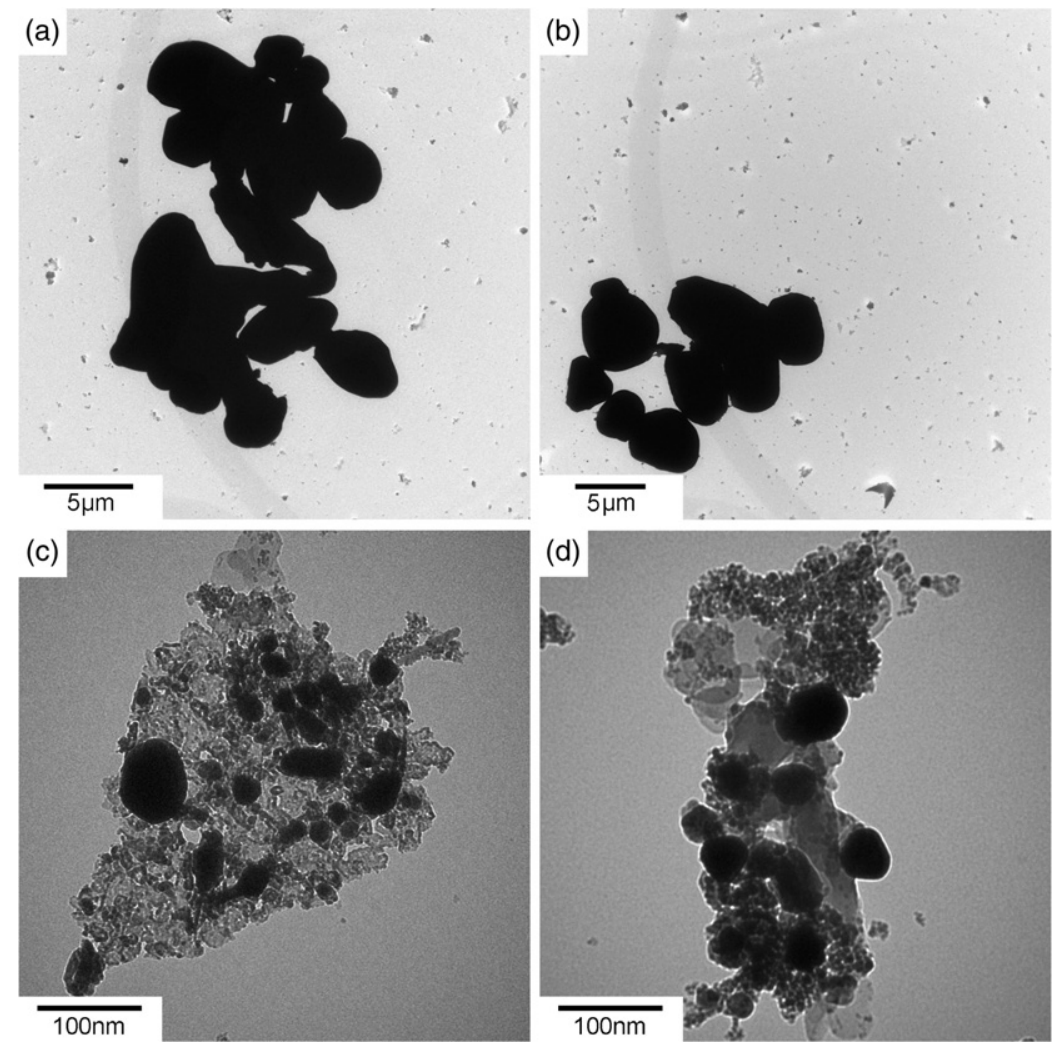

Fig. 4. TEM images of S-3 prepared in SCW for $30 \mathrm{~min}$. 
with the sizes smaller than $1 \mu \mathrm{m}$ also disappear in Fig. 4. Furthermore, compared with Fig. 3, a lot of small particles with irregular shapes can be found. As can be seen from Fig. 4(c) and (d), these small particles are composed of many Ag nanoparticles (with the sizes smaller than $100 \mathrm{~nm}$ ). However, these Ag nanoparticles aggregate together. Then Ag nanoparticles are obtained after SCW treatment.

The forming process of $\mathrm{Ag}$ nanoparticles can be explained as follows:

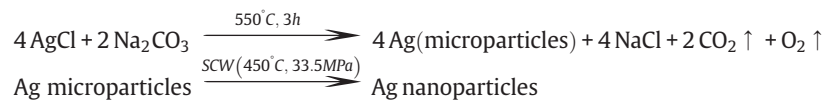

In the work, Ag microparticles (shown in Fig. 2) were previously prepared by calcination method. When Ag microparticles were treated in SCW, the large Ag microparticles with the sizes bigger than $2 \mu \mathrm{m}$ remained because of the high crystallinity. On the contrary, the small Ag microparticles with the sizes smaller than $1 \mu \mathrm{m}$ were destroyed due to the destructive effect of SCW. In this study, SCW provided a highly destructive environment. Moreover, with the increase of reaction time, the small $\mathrm{Ag}$ microparticles were destroyed further and $\mathrm{Ag}$ nanoparticles were obtained.

\section{Conclusions}

A novel approach for preparing $\mathrm{Ag}$ nanoparticles using $\mathrm{Ag}$ microparticles as precursors in SCW was found. It was believed that, the highly destructive ability of SCW could cause Ag microparticles to break down into Ag nanoparticles with regular shape and small size by optimizing the parameters such as the reaction time, temperature and pressure. In addition, the conditions of preparing $\mathrm{Ag}$ precursors also need to be optimized in the future.

\section{Acknowledgment}

This research was financially supported, in part, by the National Key Technology R\&D Program (2008BAC32B03) of China.

\section{References}

[1] Wang YH, Zhou J, Wang T. Mater Lett 2008;62:1937-40.

[2] Chen CY, Chiang CL. Mater Lett 2008;62:3607-9.

[3] Chen H, Gao F, He R, Cui DX. J Colloid Interface Sci 2007;315:158-63.

[4] Zhang ZT, Zhao B, Hu LM. J Solid State Chem 1996;121:105-10.

[5] Shin HS, Yang HJ, Kim SB, Lee MS. J Colloid Interface Sci 2004;274:89-94.

[6] Ji M, Chen XY, Wai CM, Fulton JL. J Am Chem Soc 1999;121:2631-2.

[7] Zhang WZ, Qiao XL, Chen JG. Mater Sci Eng B 2007;142:1-15.

[8] Ziegler KJ, Doty RC, Johnston KP, Korgel BA. J Am Chem Soc 2001;123:7797-803.

[9] Viswanathan R, Gupta RB. J Supercrit Fluids 2003;27:187-93.

[10] Otsu J, Oshima Y. J Supercrit Fluids 2005;33:61-7. 